(c) 2005 International Press

Adv. Theor. Math. Phys. 9 (2005) 729-747

\title{
Ghosts in the matter forms
}

\author{
Davoud Kamani \\ Institute for Studies in Theoretical Physics and Mathematics (IPM), \\ P.O. Box: 19395-5531, Tehran, Iran
}

\begin{abstract}
In this paper we study the matter form of the conformal and superconformal ghosts action. That is, the ghost fields will be expressed in terms of some scalar and spinor fields. Thus, we obtain a twodimensional covariant action in the matter form, i.e., $S_{g}$. The Poincarélike symmetries and various supersymmetries of this covariant action are analyzed. The signatures $10+2$ and $11+3$ for the total target space of the superstring theory also will be discussed.
\end{abstract}

\section{Introduction}

In the recent years, string theories can be understood by assuming the existence of higher dimensional target spaces [1-6]. The 11-dimensional M-theory [3] and the 12-dimensional F-theory [4] are examples of this context. The analysis of the super $p$-brane scanning allows spacetimes with non-Lorentzian signatures [5]. In other words, there are several models which have more than one "time" coordinate [2-6]. In particular, 12-dimensional theories and their invariances with respect to the $S O(10,2)$ rotations have been investigated [2].

Since the superstring possesses gauge symmetries, namely worldsheet reparametrization invariance, the procedure for the path integral quantization of the superstring is the Faddeev-Popov method. On the other hand,

e-print archive: http://lanl.arXiv.org/abs/hep-th/0404035 
ghosts are quantum fields used to give a functional integral representation of the Faddeev-Popov determinant [7]. They also have an important role in the BRST quantization [8]. If two- or more-time world is real, we should be able to formulate the superstring theory in the language of two- or more-time physics without the conformal and super-conformal ghosts.

We shall express the action of the super-conformal and conformal ghosts in the covariant form of the matter fields. Therefore, the ghost fields have expressions in terms of the bosonic and fermionic fields. Quantum consistency of the ghosts action in the matter form and initial form will be shown. The matter form of the action enables us to study the symmetries of the theory. Two of these symmetries are $N=1$ and $N=2$ supersymmetries. However, in these formulations the superstring lives in the $11+3$ or $10+2$ dimensional spacetimes without any ghost field.

Besides the $N=2$ supersymmetry, the theory is invariant with respect to two Poincaré-like symmetries and two other supersymmetries. For each of these symmetries there are two conserved currents. That is, each symmetry is described by the product of two distinct groups.

This paper is organized as follows. In Section 2, by introducing some vectors, the super-conformal and conformal ghosts and their action will be expressed in terms of the matter fields. In Section 3, the superstring action beyond the dimension 10 will be presented. In Section 4, Poincarélike symmetries, bi-supersymmetries and $N=2$ supersymmetry of the new form of the ghosts action will be studied. In Section 5, the signature $11+3$ for the total target spacetime of the superstring theory will be discussed.

\section{Matter form of the ghosts action}

The superstring with the worldsheet supersymmetry has the action

$$
S=-\frac{1}{4 \pi \alpha^{\prime}} \int d^{2} \sigma\left(G_{\mu \nu}\left(\eta^{a b} \partial_{a} X^{\mu} \partial_{b} X^{\nu}-i \bar{\psi}^{\mu} \rho^{a} \partial_{a} \psi^{\nu}\right)\right)+S_{g}
$$

where $S_{g}$ is sum of the conformal and super-conformal ghosts actions, i.e., $S_{g}=S_{s c g}+S_{c g}$. As we know, the spacetime corresponding to action (2.1) has the dimension 10 with the signature $9+1$. Now we proceed to study the covariant matter form of the action $S_{g}$. 


\subsection{The super-conformal ghosts}

The super-conformal ghosts have the action

$$
S_{s c g}=\frac{1}{2 \pi \alpha^{\prime}} \int d^{2} \sigma\left(\beta \partial_{+} \gamma+\tilde{\beta} \partial_{-} \tilde{\gamma}\right)
$$

where $\partial_{ \pm}=1 / 2\left(\partial_{\tau} \pm \partial_{\sigma}\right)$. In this action the fields $\beta$ and $\gamma$ (and also $\tilde{\beta}$ and $\tilde{\gamma}$ ) enter symmetrically despite their asymmetrical appearance. This is due to the flat worldsheet.

Consider the quantities $\left\{Y^{P}, \tilde{Y}^{P}, \partial_{+} Z^{P}, \partial_{-} \tilde{Z}^{P}\right\}$. We express them in terms of the variables $\left\{\partial_{-} X^{p}, \partial_{+} X^{p}, \partial_{-} \tilde{X}^{p}, \partial_{+} \tilde{X}^{p}\right\}$ as in the following

$$
\begin{aligned}
Y^{P} & =e_{p}^{P} \partial_{-} X^{p}, \\
\partial_{+} Z^{P} & =e_{p}^{P} \partial_{+} X^{p}, \\
\tilde{Y}^{P} & =e_{p}^{P} \partial_{+} \tilde{X}^{p}, \\
\partial_{-} \tilde{Z}^{P} & =e_{p}^{P} \partial_{-} \tilde{X}^{p},
\end{aligned}
$$

where the set $\left\{e_{p}^{P}\right\}$ denotes the two-dimensional vielbeins with $p, P \in\{1,2\}$. The other vielbeins (e.g., those that connect $Y^{P}$ to $\partial_{+} X^{p}$ and $\partial_{ \pm} \tilde{X}^{p}$ ) are zero. Equations (2.3) imply that only four coordinates of the set $\left\{Y^{P}, \tilde{Y}^{P}, Z^{P}, \tilde{Z}^{P}\right\}$ are independent. For example, for the constant vielbeins there are $\partial_{+} Y^{P}=\partial_{+} \partial_{-} Z^{P}$ and $\partial_{-} \tilde{Y}^{P}=\partial_{+} \partial_{-} \tilde{Z}^{P}$.

We demand action (2.2) to be

$$
S_{s c g}=\frac{1}{2 \pi \alpha^{\prime}} \int d^{2} \sigma\left(\eta_{P Q}\left(Y^{P} \partial_{+} Z^{Q}+\tilde{Y}^{P} \partial_{-} \tilde{Z}^{Q}\right)\right)
$$

where the metric $\eta_{P Q}$ has the Lorentzian signature, i.e., $\eta_{P Q}=\operatorname{diag}(-1,1)$. This action contains the bosonic fields and has the feature of action (2.2). Therefore, the fields of (2.4) have the roles of the super-conformal ghosts. Expanding action (2.4) gives two copies of action (2.2). On the other hand, action (2.4) has all symmetries of action (2.2).

Define the two-dimensional metric $G_{p q}$ as

$$
\begin{aligned}
G_{p q} & =\sum_{P, Q}\left(\eta_{P Q} e_{p}^{P} e_{q}^{Q}\right), \\
\eta^{P Q} & =\sum_{p, q}\left(G^{p q} e_{p}^{P} e_{q}^{Q}\right) .
\end{aligned}
$$


Therefore, action (2.4) takes the form

$$
S_{s c g}=-\frac{1}{8 \pi \alpha^{\prime}} \int d^{2} \sigma\left(G_{p q} \eta^{a b}\left(\partial_{a} X^{p} \partial_{b} X^{q}+\partial_{a} \tilde{X}^{p} \partial_{b} \tilde{X}^{q}\right)\right) .
$$

This action corresponds to the manifold $\mathcal{M}_{g}$ with the coordinates $\left\{X^{p}, \tilde{X}^{p}\right\}$ and the metric

$$
\bar{G}=\left(\begin{array}{cc}
\frac{1}{2} G_{p q} & 0 \\
0 & \frac{1}{2} G_{p q}
\end{array}\right) .
$$

We call $\mathcal{M}_{g}$ as the ghosts manifold. The metric $G_{p q}$ provides a background for the string which propagates in this manifold. This form of the action is covariant with respect to the worldsheet indices $\{a, b\}$ and the manifold indices $\{p, q\}$. We shall see that there are some conditions on the fields of action (2.6), which give the equality of the degrees of freedom of this action with action $(2.2)$.

In a system with the $D$-dimensional Poincaré symmetry $I S O(D-1,1)$, the conformal symmetry is $S O(D, 2)$. In fact, when one considers the conformal symmetry, the symmetry $S O(1,1)$ is added to the original global symmetry. Thus, besides the usual time coordinate, the conformal symmetry introduces another time coordinate. These imply that the ghosts manifold is product of two identical copies of a two-dimensional spacetime. In other words, we have a four-dimensional spacetime with the signature $2+2$. We shall see that the $1+1$ interpretation for the signature of this spacetime also is possible.

In fact, action (2.4) is auxiliary. Since actions (2.2) and (2.4) have the same feature, we can write

$$
\begin{aligned}
& \beta \partial_{+} \gamma=\eta_{P Q} Y^{P} \partial_{+} Z^{Q}, \\
& \tilde{\beta} \partial_{-} \tilde{\gamma}=\eta_{P Q} \tilde{Y}^{P} \partial_{-} \tilde{Z}^{Q} .
\end{aligned}
$$

Let $V^{P}$ and $\tilde{V}^{P}$ be two unit vectors, i.e.,

$$
\eta_{P Q} V^{P} V^{Q}=\eta_{P Q} \tilde{V}^{P} \tilde{V}^{Q}=1
$$

Insertion of these vectors in the left-hand side of equations (2.8) leads to the equations

$$
\begin{aligned}
& (\beta V)^{\mathrm{T}} \eta\left(V \partial_{+} \gamma\right)=Y^{\mathrm{T}} \eta \partial_{+} Z \\
& (\tilde{\beta} \tilde{V})^{\mathrm{T}} \eta\left(\tilde{V} \partial_{-} \tilde{\gamma}\right)=\tilde{Y}^{\mathrm{T}} \eta \partial_{-} \tilde{Z}
\end{aligned}
$$


One solution of these equations is

$$
\begin{aligned}
\beta V & =Y, \\
V \partial_{+} \gamma & =\partial_{+} Z+U, \\
\tilde{\beta} \tilde{V} & =\tilde{Y} \\
\tilde{V} \partial_{-} \tilde{\gamma} & =\partial_{-} \tilde{Z}+\tilde{U},
\end{aligned}
$$

where the vectors $U^{P}$ and $\tilde{U}^{P}$ are perpendicular to $Y^{P}$ and $\tilde{Y}^{P}$, respectively

$$
\eta_{P Q} Y^{P} U^{Q}=\eta_{P Q} \tilde{Y}^{P} \tilde{U}^{Q}=0
$$

However, for the next purposes, we assume they are not perpendicular to $V^{P}$ and $\tilde{V}^{P}$, i.e.,

$$
\eta_{P Q} V^{P} U^{Q} \neq 0, \quad \eta_{P Q} \tilde{V}^{P} \tilde{U}^{Q} \neq 0
$$

Other solutions for equations (2.10) are possible. For example, they can be written in the form $\left(V \partial_{+} \gamma\right)^{\mathrm{T}} \eta(\beta V)=Y^{\mathrm{T}} \eta \partial_{+} Z$ and $\left(\tilde{V} \partial_{-} \tilde{\gamma}\right)^{\mathrm{T}} \eta(\tilde{\beta} \tilde{V})=$ $\tilde{Y}^{\mathrm{T}} \eta \partial_{-} \tilde{Z}$. The solution of these equations is different from equation (2.11). We consider only solution (2.11).

In terms of the fields of action (2.4) and also in terms of the coordinates $\left\{X^{p}, \tilde{X}^{p}\right\}$, the super-conformal ghosts have the following expressions

$$
\begin{aligned}
\beta & =\eta_{P Q} V^{P} Y^{Q}=v_{p} \partial_{-} X^{p}, \\
\partial_{+} \gamma & =\eta_{P Q} V^{P}\left(\partial_{+} Z^{Q}+U^{Q}\right)=v_{p} \partial_{+} X^{p}+v \cdot u, \\
\tilde{\beta} & =\eta_{P Q} \tilde{V}^{P} \tilde{Y}^{Q}=\tilde{v}_{p} \partial_{+} \tilde{X}^{p}, \\
\partial_{-} \tilde{\gamma} & =\eta_{P Q} \tilde{V}^{P}\left(\partial_{-} \tilde{Z}^{Q}+\tilde{U}^{Q}\right)=\tilde{v}_{p} \partial_{-} \tilde{X}^{p}+\tilde{v} \cdot \tilde{u},
\end{aligned}
$$

where the vectors $v_{p}$ and $\tilde{v}_{p}$ have the definitions

$$
\begin{aligned}
& v_{p}=\eta_{P Q} e_{p}^{P} V^{Q}, \\
& \tilde{v}_{p}=\eta_{P Q} e_{p}^{P} \tilde{V}^{Q},
\end{aligned}
$$

similarly for the vectors $u_{p}$ and $\tilde{u}_{p}$. The inner product $v \cdot u$ is defined by $v \cdot u=G_{p q} v^{p} u^{q}$. According to equation (2.13), $v \cdot u$ and $\tilde{v} \cdot \tilde{u}$ are nonzero. Note that $v_{p}$ and $\tilde{v}_{p}$ also are unit vectors, i.e., $G^{p q} v_{p} v_{q}=G^{p q} \tilde{v}_{p} \tilde{v}_{q}=1$. Equations (2.14) imply that the super-conformal ghosts can be seen as linear combinations of some scalar fields.

To understand more about mappings (2.14), let us consider the bosonization of the super-ghosts $\beta$ and $\gamma$,

$$
\beta=\mathrm{e}^{-\phi} \partial_{-} \xi, \quad \gamma=\mathrm{e}^{\phi} \eta, \quad \xi=\mathrm{e}^{\zeta}, \quad \eta=\mathrm{e}^{-\zeta},
$$

where the bosonized super-ghosts are $\phi, \xi, \eta$ and $\zeta[9]$. A similar construction for the ghosts $b$ and $c$ was carried out in [10]. As we see, $\beta$ depends on the 
derivative of a field, while $\gamma$ is independent of any derivative. This also is true for $\beta$ and $\gamma$ in equations (2.14). For the field $\gamma$, also see the first equation of (2.31). Therefore, equations (2.14) can be interpreted as a kind of bosonization. Thus, the fields $X^{1}$ and $X^{2}$ have the following relations with the bosonized super-ghosts $\phi$ and $\zeta$,

$$
\begin{aligned}
v_{p} \partial_{-} X^{p} & =\mathrm{e}^{-\phi+\zeta} \partial_{-} \zeta, \\
v \cdot u+v_{p} \partial_{+} X^{p} & =\partial_{+} e^{\phi-\zeta} .
\end{aligned}
$$

Similar interpretation also holds for the left-moving fields.

According to equations (2.11), it is possible to express the fields $\left\{X^{p}, \tilde{X}^{p}\right\}$ in terms of the ghost fields

$$
\begin{aligned}
& \partial_{-} X^{p}=v^{p} \beta, \\
& \partial_{+} X^{p}=v^{p} \partial_{+} \gamma-u^{p}, \\
& \partial_{+} \tilde{X}^{p}=\tilde{v}^{p} \tilde{\beta}, \\
& \partial_{-} \tilde{X}^{p}=\tilde{v}^{p} \partial_{-} \tilde{\gamma}-\tilde{u}^{p} .
\end{aligned}
$$

The contravariant vectors are $v^{p}=e_{P}^{p} V^{P}, \tilde{v}^{p}=e_{P}^{p} \tilde{V}^{P}, u^{p}=e_{P}^{p} U^{P}$ and $\tilde{u}^{p}=e_{P}^{p} \tilde{U}^{P}$, where the matrix $e_{P}^{p}$ is inverse of the vielbein matrix $e_{p}^{P}$. These matrices satisfy the relations $e_{p}^{P} e_{P^{\prime}}^{p}=\delta_{P^{\prime}}^{P}$ and $e_{p}^{P} e_{P}{ }^{p^{\prime}}=\delta_{p}{ }^{p^{\prime}}$.

The solutions of the equations of motion of action (2.6) have the general forms $X^{p}=X_{R}^{p}+X_{L}^{p}$ and $\tilde{X}^{p}=\tilde{X}_{R}^{p}+\tilde{X}_{L}^{p}$. Half of the degrees of freedom of $X^{p}$ and $\tilde{X}^{p}$ correspond to the super-conformal ghosts. Now consider the unit vectors $V^{\prime}$ and $\tilde{V}^{\prime}$, which are perpendicular to the vectors $V$ and $\tilde{V}$, respectively. The inner products of the vectors $V^{\prime}$ and $\tilde{V}^{\prime}$ with the vectors in equations (2.11) lead to the conditions

$$
\begin{aligned}
\eta_{P Q} V^{\prime P} Y^{Q} & =v_{p}^{\prime} \partial_{-} X^{p}=0, \\
\eta_{P Q} V^{\prime P}\left(\partial_{+} Z^{Q}+U^{Q}\right) & =v_{p}^{\prime} \partial_{+} X^{p}+v^{\prime} \cdot u=0, \\
\eta_{P Q} \tilde{V}^{\prime P} \tilde{Y}^{Q} & =\tilde{v}_{p}^{\prime} \partial_{+} \tilde{X}^{p}=0, \\
\eta_{P Q} \tilde{V}^{\prime P}\left(\partial_{-} \tilde{Z}^{Q}+\tilde{U}^{Q}\right) & =\tilde{v}_{p}^{\prime} \partial_{-} \tilde{X}^{p}+\tilde{v}^{\prime} \cdot \tilde{u}=0 .
\end{aligned}
$$

These four conditions imply that the number of the degrees of freedom of actions (2.2) and (2.6) are equal. Since the vectors $v$ and $\tilde{v}$ are perpendicular to the vectors $v^{\prime}$ and $\tilde{v}^{\prime}$, respectively, these equations also can be obtained from equations (2.18). 


\subsection{The conformal ghosts}

The action of the conformal ghosts is

$$
S_{c g}=\frac{1}{2 \pi \alpha^{\prime}} \int d^{2} \sigma\left(b \partial_{+} c+\tilde{b} \partial_{-} \tilde{c}\right) .
$$

We request this action to be

$$
S_{c g}=\frac{i}{2 \pi \alpha^{\prime}} \int d^{2} \sigma\left(\eta_{P Q}\left(\Psi_{1}^{P} \partial_{+} \Theta_{1}^{Q}+\Psi_{2}^{P} \partial_{-} \Theta_{2}^{Q}\right)\right) .
$$

This action has all symmetries of action (2.20). This is due to the fact that it has two distinct copies of action (2.20).

The Grassmannian variables $\left\{\Psi_{1,2}^{P}, \Theta_{1,2}^{P}\right\}$ have the following expressions in terms of the worldsheet fermions $\left\{\psi^{p}, \theta^{p}\right\}$,

$$
\begin{aligned}
\Psi_{1}^{P} & =e_{p}^{P} \psi_{-}^{p}, \\
\Psi_{2}^{P} & =e_{p}^{P} \psi_{+}^{p}, \\
\Theta_{1}^{P} & =e_{p}^{P} \theta_{-}^{p}, \\
\Theta_{2}^{P} & =e_{p}^{P} \theta_{+}^{p} .
\end{aligned}
$$

Therefore, auxiliary action (2.21) can be written in terms of the spinor fields

$$
S_{c g}=\frac{i}{4 \pi \alpha^{\prime}} \int d^{2} \sigma\left(G_{p q} \bar{\psi}^{p} \rho^{a} \partial_{a} \theta^{q}\right)
$$

where $\psi^{p}=\left(\begin{array}{c}\psi_{-}^{p} \\ \psi_{+}^{p}\end{array}\right)$ and $\theta^{p}=\left(\begin{array}{c}\theta_{-}^{p} \\ \theta_{+}^{p}\end{array}\right)$ are Majorana spinors. We assumed that the vielbeins are independent of the fields $\left\{X^{p}, \tilde{X}^{p}\right\}$, and hence they do not depend on the worldsheet coordinates $\tau$ and $\sigma$. That is, the metric $G_{p q}$ is constant. For matching the degrees of freedom of actions (2.20), (2.21) and (2.23), see conditions (2.30).

Equality of actions (2.20) and (2.21) gives

$$
\begin{aligned}
& b \partial_{+} c=i \eta_{P Q} \Psi_{1}^{P} \partial_{+} \Theta_{1}^{Q}, \\
& \tilde{b} \partial_{-} \tilde{c}=i \eta_{P Q} \Psi_{2}^{P} \partial_{-} \Theta_{2}^{Q} .
\end{aligned}
$$

This is due to the common feature of these actions. Consider the unit vectors $W^{P}$ and $\widetilde{W}^{P}$,

$$
\eta_{P Q} W^{P} W^{Q}=\eta_{P Q} \widetilde{W}^{P} \widetilde{W}^{Q}=1 .
$$


Furthermore, define the Grassmann-valued vectors $\Lambda^{P}$ and $\tilde{\Lambda}^{P}$ with the following properties

$$
\begin{aligned}
\eta_{P Q} \Psi_{1}^{P} \Lambda^{Q} & =\eta_{P Q} \Psi_{2}^{P} \tilde{\Lambda}^{Q}=0 \\
\eta_{P Q} W^{P} \Lambda^{Q} & \neq 0, \quad \eta_{P Q} \widetilde{W}^{P} \tilde{\Lambda}^{Q} \neq 0 .
\end{aligned}
$$

Thus, for example, $\Lambda^{P}$ is perpendicular to $\Psi_{1}^{P}$, but it is not perpendicular to $W^{P}$.

Insert the unit vectors in the left-hand side of equations (2.24). Similar to relations $(2.10),(2.11)$ and $(2.14)$, we obtain the mappings

$$
\begin{aligned}
b & =i \eta_{P Q} W^{P} \Psi_{1}^{Q}=i w_{p} \psi_{-}^{p}, \\
\partial_{+} c & =\eta_{P Q} W^{P}\left(\partial_{+} \Theta_{1}^{Q}+\Lambda^{Q}\right)=w_{p} \partial_{+} \theta_{-}^{p}+w \cdot \lambda, \\
\tilde{b} & =i \eta_{P Q} \widetilde{W}^{P} \Psi_{2}^{Q}=i \tilde{w}_{p} \psi_{+}^{p}, \\
\partial_{-} \tilde{c} & =\eta_{P Q} \widetilde{W}^{P}\left(\partial_{-} \Theta_{2}^{Q}+\tilde{\Lambda}^{Q}\right)=\tilde{w}_{p} \partial_{-} \theta_{+}^{p}+\tilde{w} \cdot \tilde{\lambda} .
\end{aligned}
$$

The unit vectors $w_{p}$ and $\tilde{w}_{p}$ and the Grassmannian vectors $\lambda_{p}$ and $\tilde{\lambda}_{p}$, similar to equation (2.15), have definitions in terms of $\left\{W^{P}\right\},\left\{\widetilde{W}^{P}\right\},\left\{\Lambda^{P}\right\}$ and $\left\{\widetilde{\Lambda}^{P}\right\}$, respectively. Equation (2.27) implies that the inner products $w \cdot \lambda$ and $\tilde{w} \cdot \tilde{\lambda}$ are nonzero. Therefore, according to equations (2.28), the conformal ghosts appear as components of some spinor fields. Equivalently, the worldsheet fermions in terms of the conformal ghosts are

$$
\begin{aligned}
\psi_{-}^{p} & =-i w^{p} b, \\
\partial_{+} \theta_{-}^{p} & =w^{p} \partial_{+} c-\lambda^{p}, \\
\psi_{+}^{p} & =-i \tilde{w}^{p} \tilde{b}, \\
\partial_{-} \theta_{+}^{p} & =\tilde{w}^{p} \partial_{-} \tilde{c}-\tilde{\lambda}^{p} .
\end{aligned}
$$

Let the unit vectors $W^{\prime}$ and $\widetilde{W^{\prime}}$ be perpendicular to the vectors $W$ and $\widetilde{W}$, respectively. Therefore, we have analog of equations (2.19), i.e.,

$$
\begin{aligned}
\eta_{P Q} W^{\prime P} \Psi_{1}^{Q} & =w_{p}^{\prime} \psi_{-}^{p}=0, \\
\eta_{P Q} W^{\prime P}\left(\partial_{+} \Theta_{1}^{Q}+\Lambda^{Q}\right) & =w_{p}^{\prime} \partial_{+} \theta_{-}^{p}+w^{\prime} \cdot \lambda=0, \\
\eta_{P Q} \widetilde{W}^{\prime P} \Psi_{2}^{Q} & =\tilde{w}_{p}^{\prime} \psi_{+}^{p}=0, \\
\eta_{P Q} \widetilde{W}^{\prime P}\left(\partial_{-} \Theta_{2}^{Q}+\tilde{\Lambda}^{Q}\right) & =\tilde{w}_{p}^{\prime} \partial_{-} \theta_{+}^{p}+\tilde{w}^{\prime} \cdot \tilde{\lambda}=0 .
\end{aligned}
$$

These conditions give the same number of the degrees of freedom for actions (2.20), (2.21) and (2.23). From equations (2.29) also we can obtain these conditions. That is, use the unit vectors $w^{\prime}$ and $\tilde{w}^{\prime}$, which are perpendicular to $w$ and $\tilde{w}$, respectively. 
The physical states extracted from action (2.1) satisfy some conditions. For example, they are BRST-invariant. If we substitute the super-conformal ghosts from (2.14) and the conformal ghosts from (2.28), in the BRST charge and physical states, we obtain the equivalent BRST charge and physical states. The equivalent states under the equivalent BRST charge are invariant. This procedure also holds for other conditions on the physical states.

\subsection{Quantum consistency}

For verifying the quantization, we need the explicit forms of the fields $\gamma, \tilde{\gamma}, c$ and $\tilde{c}$. Equations (2.14) and (2.28) give them as in the following

$$
\begin{aligned}
& \gamma=v_{p} X^{p}+\gamma_{0}\left(\sigma^{-}\right)+v \cdot u \sigma^{+}, \\
& \tilde{\gamma}=\tilde{v}_{p} \tilde{X}^{p}+\tilde{\gamma}_{0}\left(\sigma^{+}\right)+\tilde{v} \cdot \tilde{u} \sigma^{-}, \\
& c=w_{p} \theta_{-}^{p}+c_{0}\left(\sigma^{-}\right)+w \cdot \lambda \sigma^{+}, \\
& \tilde{c}=\tilde{w}_{p} \theta_{+}^{p}+\tilde{c}_{0}\left(\sigma^{+}\right)+\tilde{w} \cdot \tilde{\lambda} \sigma^{-},
\end{aligned}
$$

where the vectors $\left\{v_{p}, \tilde{v}_{p}, w_{p}, \tilde{w}_{p}, u_{p}, \tilde{u}_{p}, \lambda_{p}, \tilde{\lambda}_{p}\right\}$ are considered independent of the coordinates $\sigma^{-}$and $\sigma^{+}$.

The functions $\gamma_{0}$ and $\tilde{\gamma}_{0}$ commute with all fields and $c_{0}$ and $\tilde{c}_{0}$ anticommute with all Grassmannian fields. Thus, the canonical quantization of the fields of bosonic actions (2.2) and (2.6) leads to the equations

$$
\begin{aligned}
{\left[\gamma(\tau, \sigma), \beta\left(\tau, \sigma^{\prime}\right)\right] } & =\left[\tilde{\gamma}(\tau, \sigma), \tilde{\beta}\left(\tau, \sigma^{\prime}\right)\right]=4 \pi i \alpha^{\prime} \delta\left(\sigma-\sigma^{\prime}\right) \\
{\left[X^{p}(\tau, \sigma), \partial_{\tau} X^{q}\left(\tau, \sigma^{\prime}\right)\right] } & =\left[\tilde{X}^{p}(\tau, \sigma), \partial_{\tau} \tilde{X}^{q}\left(\tau, \sigma^{\prime}\right)\right]=4 \pi i \alpha^{\prime} G^{p q} \delta\left(\sigma-\sigma^{\prime}\right) .
\end{aligned}
$$

Using equations (2.14) and (2.31) and also the equations of motion of $\gamma$ and $\tilde{\gamma}$, quantization (2.32) leads to quantization (2.33) and viceversa.

In the same way, the canonical quantization of the fields of actions (2.20) and $(2.23)$ are

$$
\begin{aligned}
\left\{c(\tau, \sigma), b\left(\tau, \sigma^{\prime}\right)\right\} & =\left\{\tilde{c}(\tau, \sigma), \tilde{b}\left(\tau, \sigma^{\prime}\right)\right\}=4 \pi i \alpha^{\prime} \delta\left(\sigma-\sigma^{\prime}\right), \\
\left\{\psi_{-}^{p}(\tau, \sigma), \theta_{-}^{q}\left(\tau, \sigma^{\prime}\right)\right\} & =\left\{\psi_{+}^{p}(\tau, \sigma), \theta_{+}^{q}\left(\tau, \sigma^{\prime}\right)\right\}=4 \pi \alpha^{\prime} G^{p q} \delta\left(\sigma-\sigma^{\prime}\right) .
\end{aligned}
$$

According to equations (2.28) and (2.31), these quantizations are the same. 


\section{$3 \quad$ Total superstring action}

Let us express the worldsheet fields $X^{p}$ and $\tilde{X}^{p}$ in terms of the new variables $x^{p}$ and $\tilde{x}^{p}$ as in the following

$$
\begin{aligned}
& X^{p}=A^{p}{ }_{q} x^{q}+\tilde{B}^{p}{ }_{q} \tilde{x}^{q}, \\
& \tilde{X}^{p}=B^{p}{ }_{q} x^{q}+\tilde{A}^{p}{ }_{q} \tilde{x}^{q} .
\end{aligned}
$$

Apply these relations in action (2.6) and then keep the cross-term of the new variables. Therefore, the matrices $A, \tilde{A}, B$ and $\tilde{B}$ should satisfy the conditions

$$
\begin{aligned}
A^{\mathrm{T}} A+B^{\mathrm{T}} B & =0, \\
\tilde{A}^{\mathrm{T}} \tilde{A}+\tilde{B}^{\mathrm{T}} \tilde{B} & =0, \\
A^{\mathrm{T}} \tilde{B}+B^{\mathrm{T}} \tilde{A} & =1 .
\end{aligned}
$$

In other words, actions (2.6) and (2.23) take the form

$$
S_{g}=-\frac{1}{4 \pi \alpha^{\prime}} \int d^{2} \sigma\left(G_{p q}\left(\eta^{a b} \partial_{a} x^{p} \partial_{b} \tilde{x}^{q}-i \bar{\psi}^{p} \rho^{a} \partial_{a} \theta^{q}\right)\right) .
$$

Obtaining action (2.6) from the bosonic part of action (3.3) gives the relations

$$
\begin{aligned}
& A \tilde{A}^{\mathrm{T}}+\tilde{B} B^{\mathrm{T}}=0, \\
& A \tilde{B}^{\mathrm{T}}+\tilde{B} A^{\mathrm{T}}=1, \\
& \tilde{A} B^{\mathrm{T}}+B \tilde{A}^{\mathrm{T}}=1 .
\end{aligned}
$$

These equations are not independent of conditions (3.2).

However, the equations of motion extracted from action (3.3) are

$$
\partial_{a} \partial^{a} x^{p}=\partial_{a} \partial^{a} \tilde{x}^{p}=\rho^{a} \partial_{a} \psi^{p}=\rho^{a} \partial_{a} \theta^{p}=0 .
$$

To remove the reparametrization invariance of action (3.3), we can write it as combination of the matter form and the ghost form

$$
\begin{aligned}
S_{g}= & -\frac{\mu}{4 \pi \alpha^{\prime}} \int d^{2} \sigma\left(G_{p q}\left(\eta^{a b} \partial_{a} x^{p} \partial_{b} \tilde{x}^{q}-i \bar{\psi}^{p} \rho^{a} \partial_{a} \theta^{q}\right)\right) \\
& +(1-\mu)\left(S_{\text {scg }}[\text { equation }(2.2)]+S_{c g}[\text { equation }(2.20)]\right),
\end{aligned}
$$

where $\mu$ is any real number. Actions in the second line do not have the diffeomorphism invariance. 
According to action (3.3), superstring action (2.1) can be written completely in the matter form

$$
S=-\frac{1}{4 \pi \alpha^{\prime}} \int d^{2} \sigma\left(G_{\bar{\mu} \bar{\nu}}\left(\eta^{a b} \partial_{a} x^{\bar{\mu}} \partial_{b} \tilde{x}^{\bar{\nu}}-i \bar{\psi}^{\bar{\mu}} \rho^{a} \partial_{a} \theta^{\bar{\nu}}\right)\right),
$$

where the metric $G_{\bar{\mu} \bar{\nu}}$ is defined by

$$
G_{\bar{\mu} \bar{\nu}}=\left(\begin{array}{cc}
G_{\mu \nu} & 0 \\
0 & G_{p q}
\end{array}\right)
$$

The coordinates $x^{\mu}$ and $\tilde{x}^{\mu}$ are defined by $x^{\mu}=\tilde{x}^{\mu}=X^{\mu}$. We also defined $\theta^{\mu}=\psi^{\mu}$. The metrics $G_{\mu \nu}$ and $G_{p q}$ represent $9+1$ and $1+1$ signatures, respectively. We shall see that the corresponding spacetime to action (3.7) can have the signatures $10+2$ and $11+3$. Now we study some symmetries of action (3.3).

\section{Symmetries of the ghosts action in the matter form}

Since the symmetries of the matter part of the total superstring action (2.1) are known, we concentrate on the symmetries of the ghost part, i.e., $S_{g}$ in form (3.3).

\subsection{The Poincaré-like symmetries}

Consider the global transformations

$$
\begin{aligned}
\delta x^{p} & =a^{p}{ }_{q} x^{q}+b^{p}, \\
\delta \tilde{x}^{p} & =\tilde{a}^{p}{ }_{q} \tilde{x}^{q}+\tilde{b}^{p}, \\
\delta \psi^{p} & =a^{p}{ }_{q} \psi^{q}, \\
\delta \theta^{p} & =\tilde{a}^{p}{ }_{q} \theta^{q},
\end{aligned}
$$

where $a_{p q}$ and $\tilde{a}_{p q}$ are antisymmetric constant matrices. Under these transformations, action (3.3), for $\tilde{a}_{p q}=a_{p q}$, is symmetric. The resulted current is

$$
J_{a}^{p q}=\frac{1}{4 \pi \alpha^{\prime}}\left[x^{p} \partial_{a} \tilde{x}^{q}-x^{q} \partial_{a} \tilde{x}^{p}+\tilde{x}^{p} \partial_{a} x^{q}-\tilde{x}^{q} \partial_{a} x^{p}+i\left(\bar{\psi}^{p} \rho_{a} \theta^{q}-\bar{\psi}^{q} \rho_{a} \theta^{p}\right)\right] .
$$

The equations of motion (3.5) imply that this is a conserved current

$$
\partial^{a} J_{a}^{p q}=0
$$


For the translations $\tilde{b}^{p}$ and $b^{p}$, the associated currents are

$$
\begin{aligned}
& P_{a}^{p}=\frac{1}{4 \pi \alpha^{\prime}} \partial_{a} x^{p}, \\
& \tilde{P}_{a}^{p}=\frac{1}{4 \pi \alpha^{\prime}} \partial_{a} \tilde{x}^{p},
\end{aligned}
$$

respectively. These currents are also conserved

$$
\partial^{a} P_{a}^{p}=\partial^{a} \tilde{P}_{a}^{p}=0
$$

For $\tilde{b}^{p}=b^{p}$, action (3.3) again remains invariant and hence we have the conserved current

$$
\mathcal{P}_{a}^{p}=\frac{1}{4 \pi \alpha^{\prime}}\left(\partial_{a} x^{p}+\partial_{a} \tilde{x}^{p}\right)
$$

Another symmetry of action (3.3) is as follows

$$
\begin{aligned}
\delta x^{p} & =a^{p}{ }_{q} \tilde{x}^{q}+b^{p}, \\
\delta \tilde{x}^{p} & =\tilde{a}^{p}{ }_{q} x^{q}+\tilde{b}^{p}, \\
\delta \psi^{p} & =a^{p}{ }_{q} \theta^{q} \\
\delta \theta^{p} & =-\tilde{a}^{p}{ }_{q} \psi^{q} .
\end{aligned}
$$

In these transformations, the parameters $a_{p q}$ and $\tilde{a}_{p q}$ can be different. Therefore, there are two types of group generators. The associated currents are

$$
\begin{aligned}
& j_{a}^{p q}=\frac{1}{2 \pi \alpha^{\prime}}\left(x^{p} \partial_{a} x^{q}-x^{q} \partial_{a} x^{p}+i \bar{\psi}^{p} \rho_{a} \psi^{q}\right), \\
& \tilde{j}_{a}^{p q}=\frac{1}{2 \pi \alpha^{\prime}}\left(\tilde{x}^{p} \partial_{a} \tilde{x}^{q}-\tilde{x}^{q} \partial_{a} \tilde{x}^{p}+i \bar{\theta}^{p} \rho_{a} \theta^{q}\right) .
\end{aligned}
$$

Note that $j_{a}^{p q}$ corresponds to the parameter $\tilde{a}_{p q}$, while $\tilde{j}_{a}^{p q}$ corresponds to $a_{p q}$. These currents also satisfy the conservation laws

$$
\partial^{a} j_{a}^{p q}=\partial^{a} \tilde{j}_{a}^{p q}=0
$$

The current equations for the translation parts are the same as equations (4.4)-(4.6). Since the equations (4.1) and (4.7) are similar to the usual Poincaré transformations, we call them as the Poincaré-like transformations. 


\subsection{Worldsheet supersymmetries}

The matter part of action (2.1) is symmetric under the worldsheet supersymmetry transformations

$$
\begin{aligned}
\delta X^{\mu} & =\bar{\epsilon} \psi^{\mu}, \\
\delta \psi^{\mu} & =-i \rho^{a} \partial_{a} X^{\mu} \epsilon,
\end{aligned}
$$

where $\epsilon$ is an infinitesimal constant spinor. Now we study the various supersymmetries of ghosts action (3.3).

\subsubsection{Bi-supersymmetries of the worldsheet}

Consider the following transformations

$$
\begin{aligned}
\delta x^{p} & =\bar{\eta} \psi^{p}, \\
\delta \tilde{x}^{p} & =\overline{\tilde{\eta}} \theta^{p}, \\
\delta \psi^{p} & =-i \rho^{a} \partial_{a} x^{p} \eta, \\
\delta \theta^{p} & =-i \rho^{a} \partial_{a} \tilde{x}^{p} \tilde{\eta},
\end{aligned}
$$

where $\eta$ and $\tilde{\eta}$ are anticommuting constant Majorana spinors. In fact, these are two independent transformations of $\left(x^{p}, \psi^{p} ; \eta\right)$ and $\left(\tilde{x}^{p}, \theta^{p} ; \tilde{\eta}\right)$. The associated supercurrents are

$$
\begin{aligned}
J_{a} & =\frac{1}{2} G_{p q} \rho^{b} \rho_{a} \psi^{p} \partial_{b} \tilde{x}^{q}, \\
\tilde{J}_{a} & =\frac{1}{2} G_{p q} \rho^{b} \rho_{a} \theta^{p} \partial_{b} x^{q} .
\end{aligned}
$$

They correspond to the parameters $\eta$ and $\tilde{\eta}$, respectively. Since there are two supercurrents, we call this symmetry as bi-supersymmetry. For $\tilde{\eta}=\eta$, action (3.3) again remains symmetric. This leads to the current

$$
\mathcal{J}_{a}=\frac{1}{2} G_{p q} \rho^{b} \rho_{a}\left(\psi^{p} \partial_{b} \tilde{x}^{q}+\theta^{p} \partial_{b} x^{q}\right) .
$$

Supercurrents (4.12) and (4.13) obey the conservation laws, i.e.,

$$
\partial^{a} J_{a}=\partial^{a} \tilde{J}_{a}=\partial^{a} \mathcal{J}_{a}=0 .
$$

Besides transformations (4.11), action (3.3) also is invariant under the following bi-supersymmetry transformations

$$
\begin{aligned}
\delta x^{p} & =\bar{\lambda} \theta^{p}, \\
\delta \tilde{x}^{p} & =\overline{\tilde{\lambda}} \psi^{p}, \\
\delta \psi^{p} & =-i \rho^{a} \partial_{a} \tilde{x}^{p} \tilde{\lambda}, \\
\delta \theta^{p} & =-i \rho^{a} \partial_{a} x^{p} \lambda,
\end{aligned}
$$


where $\lambda$ and $\tilde{\lambda}$ are infinitesimal constant real spinors. These transformations contain two independent parts $\left(x^{p}, \theta^{p} ; \lambda\right)$ and $\left(\tilde{x}^{p}, \psi^{p} ; \tilde{\lambda}\right)$. The associated supercurrents corresponding to the parameters $\lambda$ and $\tilde{\lambda}$ are

$$
\begin{aligned}
k_{a} & =\frac{1}{2} G_{p q} \rho^{b} \rho_{a} \theta^{p} \partial_{b} \tilde{x}^{q}, \\
\tilde{k}_{a} & =\frac{1}{2} G_{p q} \rho^{b} \rho_{a} \psi^{p} \partial_{b} x^{q} .
\end{aligned}
$$

For $\tilde{\lambda}=\lambda$, we obtain analog of current (4.13),

$$
\mathcal{K}_{a}=\frac{1}{2} G_{p q} \rho^{b} \rho_{a}\left(\psi^{p} \partial_{b} x^{q}+\theta^{p} \partial_{b} \tilde{x}^{q}\right) .
$$

These supercurrents satisfy the conservation equations

$$
\partial^{a} k_{a}=\partial^{a} \tilde{k}_{a}=\partial^{a} \mathcal{K}_{a}=0
$$

\subsubsection{The $N=2$ supersymmetry}

The $S O(10,2)$ covariant extension of the superstring is considered. For this, we change the worldsheet fermions $\left\{\psi^{p}, \theta^{p}\right\}$ with $\left\{\chi^{p}, \tilde{\chi}^{p}\right\}$ as in the following

$$
\begin{aligned}
\psi^{p} & =\left(\tilde{B}^{\mathrm{T}}\right)^{p}{ }_{q} \chi^{q}+\left(\tilde{A}^{\mathrm{T}}\right)^{p}{ }_{q} \tilde{\chi}^{q}, \\
\theta^{p} & =\left(A^{\mathrm{T}}\right)^{p}{ }_{q} \chi^{q}+\left(B^{\mathrm{T}}\right)^{p}{ }_{q} \tilde{\chi}^{q} .
\end{aligned}
$$

The matrices in these equations are the same that appeared in equations (3.1), (3.2) and (3.4). Now we introduce these relations in action (2.23). Therefore, according to action (2.6), the ghosts action takes the form

$$
\begin{aligned}
S_{g}= & -\frac{1}{8 \pi \alpha^{\prime}} \int d^{2} \sigma\left(G _ { p q } \left[\eta^{a b}\left(\partial_{a} X^{p} \partial_{b} X^{q}+\partial_{a} \tilde{X}^{p} \partial_{b} \tilde{X}^{q}\right)\right.\right. \\
& \left.\left.-i\left(\bar{\chi}^{p} \rho^{a} \partial_{a} \chi^{q}+\overline{\tilde{\chi}}^{p} \rho^{a} \partial_{a} \tilde{\chi}^{q}\right)\right]\right) .
\end{aligned}
$$

Obtaining the fermionic part, conditions (3.2) and (3.4) have been used.

This action manifestly describes the $N=2$ supersymmetry. The supersymmetry transformations are

$$
\begin{aligned}
\delta X^{p} & =\bar{\epsilon} \chi^{p}+\overline{\tilde{\epsilon}} \tilde{\chi}^{p}, \\
\delta \tilde{X}^{p} & =\bar{\epsilon} \tilde{\chi}^{p}-\overline{\tilde{\epsilon}} \chi^{p}, \\
\delta \chi^{p} & =-i \rho^{a} \partial_{a} X^{p} \epsilon+i \rho^{a} \partial_{a} \tilde{X}^{p} \tilde{\epsilon}, \\
\delta \tilde{\chi}^{p} & =-i \rho^{a} \partial_{a} X^{p} \tilde{\epsilon}-i \rho^{a} \partial_{a} \tilde{X}^{p} \epsilon .
\end{aligned}
$$

The worldsheet fermions $\chi^{p}$ and $\tilde{\chi}^{p}$ form an $S O(2)$ doublet.

In the appearance of the $N=2$ supersymmetry, the fields $\left\{X^{p}, \chi^{p}, \tilde{X}^{p}, \tilde{\chi}^{p}\right\}$ in the natural way appeared in action (4.20). In other words, all fields in 
this action have originated from the conformal and super-conformal ghosts. That is, they have not been introduced by hand. Obtaining worldsheet supersymmetry with $N \geq 2$, some extra fields usually are added to action by hand.

In fact, action (4.20) shows the other matter form of the ghosts action. In this manner, the manifold $\mathcal{M}_{g}$ represents two-dimensional spacetime with the coordinates $\left\{X^{p}\right\}$ or $\left\{\tilde{X}^{p}\right\}$ and the signature $1+1$. This implies that the total target space for the superstring is $10+2$-dimensional spacetime with the coordinates $\left\{X^{\mu}, X^{p}\right\}$ or $\left\{X^{\mu}, \tilde{X}^{p}\right\}$. As it was explained, this signature has origin in the conformal symmetry. On the other hand, this space is product of the 10-dimensional spacetime and the two-dimensional manifold $\mathcal{M}_{g}$. Note that one direction of development of supersymmetric theories is consideration of 12-dimensional theories with the signature 10+2 [2].

The inverse of the fermions redefinition (4.19) is

$$
\begin{aligned}
& \chi^{p}=A^{p}{ }_{q} \psi^{q}+\tilde{B}^{p}{ }_{q} \theta^{q}, \\
& \tilde{\chi}^{p}=B^{p}{ }_{q} \psi^{q}+\tilde{A}^{p}{ }_{q} \theta^{q} .
\end{aligned}
$$

Action of the operators $\partial_{ \pm}$on equations (3.1) and then comparing the resulted equations with (4.22) give the following pairs of the super-partners

$$
\begin{aligned}
& X^{p} \longleftrightarrow \chi^{p}, \\
& \tilde{X}^{p} \longleftrightarrow \tilde{\chi}^{p}, \\
& x^{p} \longleftrightarrow \psi^{p}, \\
& \tilde{x}^{p} \longleftrightarrow \theta^{p} .
\end{aligned}
$$

These are based on actions (3.3) and (4.20) and their equations of motion. That is, $\partial_{+} x^{p}\left(\partial_{-} x^{p}\right)$ acts in the same way as $\psi_{+}^{p}\left(\psi_{-}^{p}\right)$, and so on.

Note that application of transformations (4.11) and (4.15) in equations (3.1) and (4.22) does not produce transformations (4.21). In other words, transformations (4.11), (4.15) and (4.21) are independent.

\section{The signature $11+3$ for the spacetime}

Define the block diagonal metric $\mathcal{G}_{m n}$ as

$$
\mathcal{G}_{m n}=\left(\begin{array}{ccc}
G_{\mu \nu} & 0 & 0 \\
0 & \frac{1}{2} G_{p q} & 0 \\
0 & 0 & \frac{1}{2} G_{p^{\prime} q^{\prime}}
\end{array}\right) .
$$


In fact, the metrics $G_{p q}$ and $G_{p^{\prime} q^{\prime}}$ are equal. They are given by equation (2.5). Therefore, action (4.20) and the matter part of action (2.1) can be combined as in the following

$$
S=-\frac{1}{4 \pi \alpha^{\prime}} \int d^{2} \sigma\left(\mathcal{G}_{m n}\left(\eta^{a b} \partial_{a} Z^{m} \partial_{b} Z^{n}-i \bar{\Omega}^{m} \rho^{a} \partial_{a} \Omega^{n}\right)\right),
$$

where the generalized coordinates $\left\{Z^{m}\right\}$ and the extended worldsheet spinors $\left\{\Omega^{m}\right\}$ are defined by

$$
\begin{aligned}
& Z^{\mu}=X^{\mu}, \quad Z^{p}=X^{p}, \quad Z^{p^{\prime}}=\tilde{X}^{p^{\prime}}, \\
& \Omega^{\mu}=\psi^{\mu}, \quad \Omega^{p}=\chi^{p}, \quad \Omega^{p^{\prime}}=\tilde{\chi}^{p^{\prime}} .
\end{aligned}
$$

It is easy to see that this action has the $N=1$ supersymmetry. The related transformations are analog of equation (4.10), i.e.,

$$
\begin{aligned}
& \delta Z^{m}=\bar{\varepsilon} \Omega^{m}, \\
& \delta \Omega^{m}=-i \rho^{a} \partial_{a} Z^{m} \varepsilon,
\end{aligned}
$$

where the spinor $\varepsilon$ is real and constant.

As we explained, the metrics $G_{p q}$ and $G_{p^{\prime} q^{\prime}}$ are equal. For each of the metrics $G_{p q}$ and $G_{p^{\prime} q^{\prime}}$, the symmetry $S O(1,1)$ is added to the global Poincaré symmetry. In other words, the conformal symmetry implies that the block diagonal metric $\mathcal{G}_{m n}$ and the coordinates $\left\{Z^{m}\right\}$ describe 14-dimensional spacetime with the signature $11+3$. In fact, the conformal symmetry of the system introduces the extra coordinates.

The line element of the spacetime associated to action (5.2) is

$$
d s^{2}=\mathcal{G}_{m n} d Z^{m} d Z^{n}=G_{\mu \nu} d X^{\mu} d X^{\nu}+\frac{1}{2} G_{p q}\left(d X^{p} d X^{q}+d \tilde{X}^{p} d \tilde{X}^{q}\right) .
$$

On the other hand, this spacetime is product of the two manifolds $M \times \mathcal{M}_{g}$, where $M$ is the $9+1$-dimensional spacetime and $\mathcal{M}_{g}$ is 2+2-dimensional ghosts manifold.

Note that the $N=1$ supersymmetry in $11+3$ dimensions from the various points of view has been studied. For example, see the [6].

\section{Conclusions}

The super-conformal and conformal ghosts action, i.e., $S_{g}$, was expressed in the covariant matter form. The ghost fields also were expressed in terms of the matter fields. In other words, the conformal ghosts are equivalent to some spinor fields and the super-conformal ghosts also can be represented 
by some scalar fields. We showed that the quantization of the action $S_{g}$ in the matter form and in the ghost form are consistent as expected.

We observed that the bosonic fields of the matter form of the action can be interpreted as additional coordinates of the spacetime. The manifold of these extra dimensions is $1+1$ - or 2+2-dimensional spacetime. Therefore, the total superstring action corresponds to a 12- or 14-dimensional spacetime with two and three time-directions, respectively.

We studied some symmetries of $S_{g}$ in the matter form. Under two different transformations, which are similar to the Poincaré transformations, the action is invariant. Each of these symmetries gives the various conserved currents. Furthermore, for this action, there are two different bi-supersymmetries. A bi-supersymmetry contains two parameters for the transformations and hence two conserved supercurrents. In addition, we observed that the theory has the $N=2$ supersymmetry. Finally, we obtained the total superstring action, with the $N=1$ supersymmetry, in the $11+3$-dimensional spacetime.

\section{References}

[1] E. Witten, String Theory Dynamics In Various Dimensions, Nucl. Phys. B443 (1995), 85, hep-th/9503124;

J. Dai, R.G. Leigh and J. Polchinski, New connections between string theories, Mod. Phys. Lett. A4 (1989), 2073;

M.J. Duff, J.T. Liu and R. Minasian, Eleven Dimensional Origin of String/String Duality: A One Loop Test, Nucl. Phys. B452 (1995), 261, hep-th/9506126;

S. Kar, J. Maharana and S. Panda, Dualities in Five Dimensions and Charged String Solutions, Nucl. Phys. B465 (1996), 439, hepth/9511213;

M.J. Duff, R. Minasian and E. Witten, Evidence for Heterotic/ Heterotic Duality, Nucl. Phys. B465 (1996), 413, hep-th/9601036;

C.M. Hull, String Dynamics at Strong Coupling, Nucl. Phys. B468 (1996), 113, hep-th/9512181;

N. Ohta, Phys. Rev. D33 (1986), 1681;

T. Tsukioka and Y. Watabiki, Quantization of Bosonic String Model in 26+2-dimensional Spacetime, Int. J. Mod. Phys. A19 (2004), 1923, hep-th/0308206;

A.A. Deriglazov, Bosonic sector of $D=11$ superstring action and the critical dimension. Toy model, Phys. Lett. B486 (2000), 218, hepth/0009078; 
A.A. Deriglazov, String action with multiplet of $\Theta$-terms and the hidden Poincare symmetry, Nucl. Phys. B597 (2001), 299, hep-th/0006032;

A. Popov, Phys. Lett. B259 (1991), 256.

[2] C.M. Hull, Duality and the Signature of Space-Time, JHEP 9811 (1998), 017, hep-th/9807127;

R. Manvelyan, A. Melikyan and R. Mkrtchyan, Representations and BPS states of $10+2$ superalgebra, Mod. Phys. Lett. A13 (1998), 2147, hep-th/9805148;

S.V. Ketov, Do the critical $(2,2)$ strings know about a supergravity in $2+10$ dimensions?, hep-th/9710086;

T. Ueno, BPS States in $10+2$ Dimensions, JHEP 0012 (2000), 006, hep-th/9909007;

R. Manvelyan and R. Mkrtchyan, Towards SO(2,10)-Invariant MTheory: Multilagrangian Fields, Mod. Phys. Lett. A15 (2000), 747, hep-th/9907011;

S.F. Hewson and M.J. Perry, The twelve dimensional super (2+2)brane, Nucl. Phys. B492 (1997), 249, hep-th/9612008;

S.F. Hewson, On supergravity in $(10,2)$, hep-th/9908209,

S.F. Hewson, Threebranes in twelve dimensions, hep-th/9801029;

I. Bars, S-Theory, Phys. Rev. D55 (1997), 2373, hep-th/9607112;

I. Bars, Black hole entropy reveals a 12th "dimension", Phys. Rev. D55 (1997), 3633, hep-th/9610074;

I. Bars, Two-Time Physics, hep-th/9809034;

I. Bars and C. Kounnas, String and Particle with Two Times, Phys. Rev. D56 (1997), 3664, hep-th/9705205;

I. Bars and C. Deliduman, Superstrings with new supersymmetry in $(9,2)$ and $(10,2)$ dimensions, Phys. Rev. D56 (1997), 6579, hepth/9707215;

H. Nishino, $N=2$ Chiral Supergravity in $(10+2)$-Dimensions As Consistent Background for Super $(2+2)$-Brane, Phys. Lett. B437 (1998), 303, hep-th/9706148;

H. Nishino, Supergravity in $10+2$ Dimensions as Consistent Background for Superstring, Phys. Lett. B428 (1998), 85, hep-th/9703214; H. Nishino and E. Sezgin, Supersymmetric Yang-Mills Equations in $10+2$ Dimensions, Phys. Lett. B388 (1996), 569, hep-th/9607185;

A. Tseytlin, Type IIB instanton as a wave in twelve dimensions, Phys. Rev. Lett. 78 (1997), 1864, hep-th/9612164;

S. Kar, D-Branes and Twelve Dimensions, Nucl. Phys. B497 (1997), 110, hep-th/9701117;

Y. Watabiki, The bosonic string and superstring models in $26+2$ and $10+2$ dimensional space-time, and the generalized Chern-Simons action, JHEP 0305 (2003), 001, hep-th/0303045; 
D. Kamani, PP-Wave Strings from Membrane and from String in the Spacetime with Two Time Directions, Phys. Lett. B564 (2003), 123, hep-th/0304236.

[3] J.H. Schwarz, An SL(2,Z) Multiplet of Type IIB Superstrings, Phys. Lett. B360 (1995), 13, hep-th/9508143;

J.H. Schwarz, The Power of M Theory, Phys. Lett. B367 (1996), 97, hep-th/9510086;

P.K. Townsend, Membrane tension and manifest IIB S-duality, Phys. Lett. B409 (1997), 131, hep-th/9705160;

P.K. Townsend, M-theory from its superalgebra, hep-th/9712004;

P.K. Townsend, Four Lectures on M-theory, hep-th/9612121;

E. Cremmer, B. Julia and J. Scherk, Supergravity theory in eleven dimensions, Phys. Lett. B76 (1978), 409;

E. Cremmer and B. Julia, The SO(8) supergravity, Nucl. Phys. B159 (1979), 141.

[4] C. Vafa, Evidence for F-Theory, Nucl. Phys. B469 (1996), 403, hepth/9602022.

[5] M. Blencowe and M.J. Duff, Supermembranes and the signature of space-time, Nucl. Phys. B310 (1988), 387.

[6] I. Rudychev and E. Sezgin, Superparticles in $D>11$, Phys. Lett. B415 (1997), 363, hep-th/9704057;

E. Sezgin, Super Yang-Mills in (11,3) Dimensions, Phys. Lett. B403 (1997), 265, hep-th/9703123;

I. Rudychev, E. Sezgin and P. Sundell, Supersymmetry in Dimensions Beyond Eleven, Nucl. Phys. Proc. Suppl. 68 (1998), 285, hepth/9711127;

I. Bars, A case for 14 dimensions, Phys. Lett. B403 (1997), 257, hepth/9704054.

[7] L.D. Faddeev and V.N. Popov, Feynman diagrams for the Yang-Mills field, Phys. Lett. B25 (1967), 29.

[8] C. Becchi, A. Rouet and R. Stora, The Abelian Higgs-Kibble model, unitarity of the S-operator, Phys. Lett. B52 (1974), 344;

C. Becchi, A. Rouet and R. Stora, Ann. Phys. 98 (1976), 287;

M. Kato and K. Ogawa, Covariant quantization of string based on BRST invariance, Nucl. Phys. B212 (1983), 443.

[9] D. Friedan, E. Martinec and S. Shenker, Conformal invariance, supersymmetry, and string theory, Nucl. Phys. B271 (1986), 93.

[10] C.G. Callan, C. Lovelace, C.R. Nappi and S.A. Yost, Adding holes and crosscaps to the superstring, Nucl. Phys. B293 (1987), 83. 
